\title{
Economias de Escala na Produção de Frangos de Corte no Brasil ${ }^{1}$
}

\author{
Luís Alberto Ferreira Garcia* \\ Joaquim Bento de Souza Ferreira Filho**
}

Resumo: Neste trabalho, estima-se os parâmetros de uma função de custos translog, que se mostrou adequada para representar a estrutura de custos do setor de produção de frangos de corte no Brasil. Os coeficientes encontrados foram utilizados para calcular as economias de escala no setor. Os dados do estudo são de corte transversal, obtidos a partir de pesquisa de campo em uma amostra de 229 avicultores nos principais estados produtores do país. No trabalho são apresentados, também, alguns resultados relativos às características das propriedades da amostra pesquisada, que permitem inferir sobre os resultados encontrados. As estimativas de economias de escala obtidas para o modelo de custos translog apontam uma escala ótima de produção de aproximadamente 110 mil quilos de frango vivo ou $3.500 \mathrm{~m}^{2}$ de aviário. Conclui-se que o tamanho ótimo da exploração, obtido no trabalho empírico, é menor que o sugerido por algumas

\footnotetext{
*Doutor em Economia Aplicada pela ESALQ/USP. Professor Adjunto do Colegiado de Ciências Econômicas do Centro de Ciências Sociais Aplicadas da UNIOESTE: Campus de Cascavel/PR. Rua: Terra Roxa, 1425 - Casa: 205. Região do Lago II. Cascavel/PR. CEP: 85.816-360. E-mail: sgarcia@adslcertto.com.br; ferreira@unioeste.br.

**Pós-Doutor pela Monash University, Austrália. Livre Docente do Departamento de Economia, Administração e Sociologia da ESALQ/USP. Av. Pádua Dias, 11. Piracicaba/SP. CEP: 13.418-900. E-mail: jbsferr@carpa.ciagri.usp.br.

${ }^{1} \mathrm{O}$ presente artigo baseia-se, em parte, na Tese de Doutorado do primeiro autor, apresentada ao Programa de Pós-Graduação em Economia Aplicada da ESALQ/USP, sob orientação do segundo autor.
} 
empresas de abate do setor. Estas propriedades, com produções superiores, estariam operando com deseconomias, o que poderia ocasionar uma certa pressão dos produtores por uma melhor remuneração da atividade. As evidências sugerem, também, que algumas características da pequena produção podem minimizar a importância destas economias.

Palavras-Chaves: Frangos de Corte, Função Custo Translog, Economias de Escala, Dualidade.

\section{Classificação JEL: D24, Q12}

Abstract: In this paper we estimate the parameters of a translog cost function for the broiler production activity in Brazil, to infer about scale economies in the activity. Cross-section data is used, from a survey of 229 producers in the main producing regions. Other characteristics of the producers surveyed are also discussed. Our estimates of scale economies show an optimal scale of production around 110 thousand kilos of live hens, corresponding to a 3,500 $\mathrm{m}^{2}$ plant area. The paper points to the conclusion that the optimal size of exploitation is smaller than that suggested by some processing industries. Larger scale farms would be operating with diseconomies of scale, what could generate pressures for price increases in the future. The evidence suggests also that some smallscale producers characteristics could help minimize the importance of operating under optimal scale.

Keywords: Broilers, Translog Cost Function, Economies of Scale, Duality.

JEL Classification: D24, Q12.

\section{Introdução}

A fim de atender uma demanda interna e externa crescente, a produção de frangos de corte, de excepcional desempenho no agronegócio brasileiro, tem se expandido, nos últimos anos, para outros estados do país além dos tradicionais produtores.

Com a reorganização da base agroindustrial desta cadeia produtiva, 
principalmente através da implantação de modernos projetos avícolas nos estados da região Centro-Oeste, surge um novo produtor integrado ao sistema, com perfil diferenciado. Nos estados da região Sul, principais produtores e exportadores de frango do país, a produção se deu basicamente em pequenas propriedades e em pequena escala. Por outro lado, a produção em parceria com algumas agroindústrias de abate, instaladas no Centro-Oeste brasileiro, passa a incorporar produtores com áreas de terra maiores e com escalas mais significativas de produção, tornando possível que a capacidade de abate das empresas seja suprida com um número menor de grandes avicultores.

Justifica-se, assim, a investigação empírica para determinar o tamanho ótimo da atividade de produção de frangos de corte, a fim de inferir sobre a existência ou não de economias de escala no setor. Os índices de economias de escala são obtidos a partir das estimativas dos parâmetros de uma função de custos transcendental logarítmica (translog). ${ }^{2}$

Adicionalmente, com base nos resultados encontrados para a amostra, procura-se avaliar a possibilidade dos pequenos avicultores das regiões tradicionais serem afetados, diante da concorrência com a produção avícola em grande escala do Centro-Oeste brasileiro.

\section{Referencial teórico e metodológico ${ }^{3}$}

O referencial teórico deste estudo é o da teoria microeconômica dos custos de produção e dualidade. Binswanger (1974), Lerda (1979), Silberberg (1990), Chambers (1994), Alves (1996), Barbosa (1995) e Bairam (1998),

\footnotetext{
${ }^{2} \mathrm{Na}$ verdade, a função custo nos fornece informações a respeito das "economias de tamanho" e não necessariamente "economias de escala". Apesar do uso indiscriminado na literatura, existe uma diferença significativa entre esses dois conceitos (Chambers, 1994, p.69-73). As economias de tamanho incluem as economias de escala e "outras economias" (como por exemplo, as de ordem pecuniária e de aprendizagem) que possibilitem as firmas operarem com o menor custo possível. Nada obstante, como observa o autor, apesar das diferenças, as economias de tamanho e de escala são equivalentes quando a empresa está no seu ponto de mínimo custo de produção. Dado que, no presente estudo, pressupõe-se que os produtores sejam minimizadores de custos, não se faz necessária a distinção entre estes dois conceitos. Utilizou-se o termo "economias de escala" por ser de uso mais corrente na literatura.

${ }^{3}$ Para maiores detalhes quanto aos procedimentos metodológicos ver: Garcia (2004).
} 
entre outros, são referências para função custo e dualidade. Para estimarse economias de escala na produção de frangos de corte os princípios da "teoria da dualidade" são de grande importância, ao permitir obter-se estimativas destas economias a partir da função custo, sem a necessidade de se conhecer diretamente a função de produção a ela associada.

\subsection{A função custo transcendental logarítmica - Translog}

A forma transcendental logarítmica (translog) da função custo é uma forma funcional flexível, não impondo restrições a priori à função de produção a ela associada. Segundo Binswanger (1974), as funções de custo translog são atraentes por apresentarem equações de demanda derivadas por fatores de produção lineares nos logaritmos. Para Christensen \& Greene (1976), a forma funcional da função de custos translog é importante devido ao fato de não impor nenhuma condição às possibilidades de substituição entre os fatores de produção e de admitir variações nas economias de escala de acordo com o nível de produção, característica essencial para que a curva de custo médio tenha a forma de "U” clássica.

\subsection{Descrição das variáveis}

Os dados utilizados são de corte transversal (cross section), coletados a partir da pesquisa realizada de setembro a dezembro de $2002^{4}$, em uma amostra de 229 produtores que mantém contratos de parceria com 15 empresas avícolas brasileiras nos estados do Rio Grande do Sul, Santa Catarina, Paraná, Minas Gerais (região tradicional), Goiás, Mato Grosso e Mato Grosso do Sul (região de expansão). ${ }^{5}$ A metodologia adotada para determinação

\footnotetext{
${ }^{4}$ O levantamento de campo fez parte de pesquisa mais ampla, intitulada: "Policy, techinical and environmental determinants and implications of the scalingup swine, broiler, layer and milk production in Brazil”, realizada pelo Centro de Estudos Avançados em Economia Aplicada da ESALQ/USP em parceria com a FAO/IFPRI (Food and Agricultural Organization e International Food Policy Research Institute).

${ }^{5}$ Apesar da relevante posição que ocupa o Estado de São Paulo como produtor e exportador de frango de corte no Brasil, o mesmo foi excluído da presente pesquisa pelos órgãos financiadores. Além disto, como observa Martins (2002, p. 74): “Já faz alguns anos que a avicultura paulista vem apresentando perda relativa de participação na produção nacional de carne de frango...”.
} 
dos itens constantes da planilha de custos é, basicamente, a descrita em Hoffmann (1987) e Buarque (1991), com as adaptações realizadas para o setor, descritas nos trabalhos de Canever (1997) e Ferreira (1998).

A análise dos custos de produção foi feita a partir do recebimento dos pintos de um dia pelo produtor até a entrega dos frangos vivos para a agroindústria. Deve-se observar, no entanto, que como custos do produtor parceiro da agroindústria são considerados apenas aqueles que efetivamente são de sua responsabilidade. Ou seja, não são considerados os custos que, por força do contrato de parceria avícola, são de responsabilidade da agroindústria como, por exemplo, os pintos de um dia, a ração, os medicamentos, a assistência técnica e outros.

Os custos considerados foram: capital (K), energia (E), mão-de-obra (L) e cama de frango (F), que representam aproximadamente $97 \%$ dos custos do produtor. As parcelas de custo dos fatores de produção selecionados foram calculadas dividindo-se as despesas totais com cada um deles pelo custo total da atividade. Os preços dos fatores foram obtidos diretamente, através da pesquisa, ou pela razão entre a despesa com o fator e a quantidade utilizada do mesmo.

Os dados apresentados, relativos às características das propriedades nas diferentes regiões produtoras, foram obtidos, também, a partir dos resultados da pesquisa de campo realizada com os produtores da amostra selecionada.

\subsection{Procedimentos de estimação}

O procedimento de estimação utilizado segue o descrito em Greene (2000, p.630 a 644), calculando-se conjuntamente a função custo total translog e as equações de parcelas de custo como um sistema de "regressão multivariada”. Segundo o autor, a estimativa conjunta das funções de custo total translog e de parcelas de custo, resultam em parâmetros mais eficientes do que aqueles que seriam obtidos aplicando-se Mínimos Quadrados Ordinários (M.Q.O.) para a função custo sozinha. Esta metodologia foi utilizada em trabalhos como os de Christensen \& Greene (1976), Ray (1982) e Albuquerque (1987), entre outros. Assume-se que os erros têm distribuição normal e são aditivos para cada uma das equações de parcela de custos e para a função custo. 
Para tornar o modelo operacional, impõem-se as restrições teóricas de simetria e homogeneidade através da normalização da função custo total translog. O problema da singularidade da matriz de variância e covariância dos erros das equações de parcela de custos é equacionado deixando-se de fora a equação de parcela de custos de um dos fatores de produção. Estima-se, assim, o restante do sistema e obtêm-se os parâmetros excluídos por diferença. Em princípio, é irrelevante qual o fator é escolhido como aquele a ser excluído. Segundo Greene (2000), os parâmetros obtidos serão invariantes em relação à equação a ser suprimida deixando-se interagir as estimativas por Mínimos Quadrados Generalizados (M.Q.G.) ou estimando-se o modelo por Máxima Verossimilhança. Neste sentido, computam-se estimativas de Máxima Verossimilhança dos parâmetros para assegurar a invariância com respeito à escolha da equação de parcela de custos a ser deixada de fora do modelo.

Considerando-se as estimativas do modelo obtidas por Máxima Verossimilhança, pode-se testar as hipóteses de homoteticidade, homogeneidade e de elasticidade de substituição unitária da função de produção, usando-se o teste de "razão de verossimilhança" (likelihood ratio test). Especificando-se os determinantes das estimativas restritas (R) e irrestritas (U) da matriz de variâncias e covariâncias dos erros por $\Omega_{\mathrm{R}}$ e $\Omega_{\mathrm{U}}$, respectivamente, pode-se definir a relação de probabilidade como sendo: (Christensen \& Greene, 1976 e Greene, 2000).

$$
\lambda=\left(\frac{\left(\left|\hat{\Omega}_{R}\right|\right)}{\left(\left|\hat{\Omega}_{U}\right|\right)}\right)^{-T / 2}
$$

Onde $T$ é o número de firmas (observações).

Testa-se estas hipóteses usando-se o fato que $-2 \ln \lambda$ é assintoticamente distribuído com distribuição de qui-quadrado com graus de liberdade iguais ao número de restrições independentes que são impostas.

\subsection{Estimativa do modelo}

Define-se $i$ e $j$ como os fatores de produção capital (K), energia (E), mão-de-obra (L) e cama de frango (F). $S_{K}, S_{E}, S_{L}$ e $S_{F}$ referem-se 
às parcelas de gastos com capital, energia, trabalho e cama de frango respectivamente. $Y$ é o valor da produção de frangos vivos (em kg). $P_{K}$ $P_{E}, P_{L}, P_{F}$ são os preços dos insumos (em $R$ ).

As restrições de homogeneidade linear e as de simetria, são incorporadas ao sistema através da normalização das equações pelo preço do insumo cama de frango $(\mathrm{F})$.

Como a soma das parcelas dos gastos com os fatores capital, energia, trabalho e cama de frango é igual à unidade $\left(S_{K}+S_{E}+S_{L}+S_{F}=1\right)$, suprime-se a equação da parcela de gastos com cama de frango para evitar uma matriz de covariância singular. Desta forma, o modelo a ser estimado, por Máxima Verossimilhança, com as restrições impostas, fica composto do seguinte conjunto de equações:

$$
\begin{aligned}
& \ln \left(C^{*} P_{F}\right)=\beta_{0}+\beta_{Y} \ln Y+\beta_{K} \ln \left(P_{K} / P_{F}\right)+\beta_{E} \ln \left(P_{E} / P_{F}\right)+\beta_{L} \ln \left(P_{L} / P_{F}\right)+ \\
& 1 / 2 \gamma_{K K}\left(\ln \left(P_{K} / P_{F}\right)\right)^{2}+\gamma_{K E} \ln \left(P_{K} / P_{F}\right) \ln \left(P_{E} / P_{F}\right)+\gamma_{K L} \ln \left(P_{K} / P_{F}\right) \ln \left(P_{L} / P_{F}\right)+ \\
& \gamma_{E E}\left(\ln \left(P_{E} / P_{F}\right)\right)^{2}+\gamma_{E L} \ln \left(P_{E} / P_{F}\right) \ln \left(P_{L} / P_{F}\right)++\gamma_{L L}\left(\ln \left(P_{L} / P_{F}\right)\right)^{2}+ \\
& \gamma_{K Y} \ln \left(P_{K} / P_{F}\right) \ln Y+\gamma_{E Y} \ln \left(P_{E} / P_{F}\right) \ln Y+\gamma_{L Y} \ln \left(P_{L} / P_{F}\right) \ln Y+1 / 2 \beta_{Y Y}(\ln Y)^{2} \\
& S_{K}=\beta_{K}+\gamma_{K K} \ln \left(P_{K} / P_{F}\right)+\gamma_{K E} \ln \left(P_{E} / P_{F}\right)+\gamma_{K L} \ln \left(P_{L} / P_{F}\right)+\gamma_{K Y} \ln Y \\
& S_{E}=\beta_{E}+\gamma_{K E} \ln \left(P_{K} / P_{F}\right)+\gamma_{E E} \ln \left(P_{E} / P_{F}\right)+\gamma_{E L} \ln \left(P_{L} / P_{F}\right)+\gamma_{E Y} \ln Y \\
& S_{L}=\beta_{L}+\gamma_{K L} \ln \left(P_{K} / P_{F}\right)+\gamma_{E L} \ln \left(P_{E} / P_{F}\right)+\gamma_{L L} \ln \left(P_{L} / P_{F}\right)+\gamma_{L Y} \ln Y
\end{aligned}
$$

Os parâmetros restantes são calculados por diferença, de acordo com as expressões:

$$
\begin{aligned}
& \beta_{F}=1-\beta_{K}-\beta_{E}-\beta_{L} \\
& \gamma_{F Y}=-\gamma_{K Y}-\gamma_{E Y}-\gamma_{L Y} \\
& \gamma_{K F}=-\gamma_{K K}-\gamma_{K E}-\gamma_{K L} \\
& \gamma_{E F}=-\gamma_{K E}-\gamma_{E E}-\gamma_{E L} \\
& \gamma_{L F}=-\gamma_{K L}-\gamma_{E L}-\gamma_{L L} \\
& \gamma_{F F}=-\gamma_{K F}-\gamma_{E F}-\gamma_{L F}
\end{aligned}
$$




\section{Resultados e discussão}

\subsection{Parâmetros estimados}

Economias de escala podem ser convenientemente medidas com o modelo de custo transcendental logarítmico. A diferenciação da função custo translog resulta na estimação direta da expressão da elasticidade de custos em relação à produção $\left(E C^{*}\right)$. A partir deste resultado, obtém-se os índices de economia de escala (IES) subtraindo-se $E C^{*}$ da unidade.

Considera-se, no estudo, além do modelo de custos translog (modelo A) mais quatro modelos: O modelo B impõe homoteticidade à função de produção associada; o modelo $\mathrm{C}$, homogeneidade; o modelo D impõe elasticidade de substituição unitária e o modelo E, homogeneidade e elasticidade de substituição unitária. As fórmulas para os índices de economias de escala (IES), para cada modelo são apresentadas na Tabela 1.

Tabela 1: Índices de economias de escala (IES) para os modelos de A a E

\begin{tabular}{ll}
\hline IES (A) & $1-\left(\beta_{Y}+\beta_{Y Y} \ln Y+\gamma_{K Y} \ln P_{K}+\gamma_{E Y} \ln P_{E}+\gamma_{L Y} \ln P_{L}+\gamma_{F Y} \ln P_{F}\right)$ \\
IES (B) & $1-\left(\beta_{Y}+\beta_{Y Y} \ln Y\right)$ \\
IES (C) & $1-\beta_{Y}$ \\
IEC (D) & $1-\left(\beta_{Y}+\beta_{Y Y} \ln Y+\gamma_{K Y} \ln P_{K}+\gamma_{E Y} \ln P_{E}+\gamma_{L Y} \ln P_{L}+\gamma_{F Y} \ln P_{F}\right)$ \\
IEC (E) & $1-\beta_{Y}$ \\
\hline
\end{tabular}

Fonte: baseada em Christensen \& Greene (1976).

Na Tabela 2 apresentam-se os parâmetros obtidos, por máxima verossimilhança, para os cinco modelos considerados. O modelo completo, translog (A), resultou na estimativa direta de 15 coeficientes, 13 deles estatisticamente diferentes de zero ao nível de significância de $1 \%$ e 2 deles ao nível de significância de $20 \%$. Através da imposição das restrições de simetria e homogeneidade, foram calculados os 6 (seis) parâmetros restantes do modelo sendo, 4 significativos a $1 \%$, 1 a $10 \%$ e 1 a $20 \%$. 
Tabela 2: Coeficientes da função custos para os modelos de A a E.

\begin{tabular}{|c|c|c|c|c|c|c|}
\hline \multirow{2}{*}{ Parâmetros } & \multirow{2}{*}{ Origem } & \multicolumn{5}{|c|}{ Modelo } \\
\hline & & A & $\mathrm{B}$ & $\mathrm{C}$ & $\mathrm{D}$ & $\mathrm{E}$ \\
\hline$\beta_{0}$ & regressão & $\begin{array}{c}16,724^{* * * * *} \\
(8,610)\end{array}$ & $\begin{array}{c}19,499^{* * * * *} \\
(10,431)\end{array}$ & $\begin{array}{l}1,899^{* *+4} \\
(6,642)\end{array}$ & $\begin{array}{c}15,423^{* * * *} \\
(7,0280)\end{array}$ & $\begin{array}{l}1,999^{*++\ldots+} \\
(10,122)\end{array}$ \\
\hline$\beta_{K}$ & regressão & $\begin{array}{l}-0,0657^{*} \\
(-1,325)\end{array}$ & $\begin{array}{l}0,2274^{*} \\
(3,434)\end{array}$ & $\begin{array}{c}0,2478^{* * * *} \\
(3,727)\end{array}$ & $\begin{array}{l}-0,1297^{* * * *} \\
(-2,0240)\end{array}$ & $\begin{array}{c}0,4102^{* * * * *} \\
(83,289)\end{array}$ \\
\hline$\beta_{E}$ & regressão & $\begin{array}{l}0,0515^{\circ} \\
(1,289)\end{array}$ & $\begin{array}{c}0,2325^{* * * *} \\
(6,840)\end{array}$ & $\begin{array}{c}0,2319^{* * * * *} \\
(6,910)\end{array}$ & $\begin{array}{c}-0,1881^{* * * *} \\
(-2,5490)\end{array}$ & $\begin{array}{c}0,1634^{*+\cdots * *} \\
(32,701)\end{array}$ \\
\hline$\beta_{L}$ & regressão & $\begin{array}{c}0,7975^{* * * *} \\
(11,697)\end{array}$ & $\begin{array}{c}0,2609^{* \ldots * *} \\
(4,006)\end{array}$ & $\begin{array}{c}0,2485^{* \ldots * *} \\
(3,753)\end{array}$ & $\begin{array}{l}1,1774^{* * * *} \\
(16,0650)\end{array}$ & $\begin{array}{c}0,3408^{* * * 4} \\
(56,83)\end{array}$ \\
\hline$\beta_{F}$ & $1-\beta_{K}-\beta_{E}-\beta_{L}$ & $\begin{array}{c}0,2169^{* *+*} \\
(3,814)\end{array}$ & $\begin{array}{c}0,2792^{* * * *} \\
(7,166)\end{array}$ & $\begin{array}{c}0,2719^{* * * *} \\
(6,934)\end{array}$ & $\begin{array}{l}0,1404^{* * * *} \\
(2,5410)\end{array}$ & $\begin{array}{c}0,0856^{* * * *} \\
(23,145)\end{array}$ \\
\hline$\beta_{Y}$ & regressão & $\begin{array}{c}-2,2582^{* * * * *} \\
(-6,387)\end{array}$ & $\begin{array}{c}-2,5401^{* * * *} \\
(-7,404)\end{array}$ & $\begin{array}{c}0,7144^{* * 4 *} \\
(42,166)\end{array}$ & $\begin{array}{c}-2,0915^{* * * *} \\
(-5,2200)\end{array}$ & $\begin{array}{c}0,7518^{* * * *} \\
(41,039)\end{array}$ \\
\hline$\gamma_{K Y}$ & regressão & $\begin{array}{c}0,0699^{* * * *} \\
(10,471)\end{array}$ & $\cdots \cdot$ & $\cdot \cdot \cdot$ & $\begin{array}{c}0,0506^{* * * *} \\
(8,4610)\end{array}$ & $\cdot \cdot$ \\
\hline$\gamma_{E Y}$ & regressão & $\begin{array}{l}0,0171^{* * * *} \\
(2,732)\end{array}$ & $\cdots$ & & $\begin{array}{c}0,0329^{* \ldots *} \\
(4,7660)\end{array}$ & $\cdots \cdot$ \\
\hline$\gamma_{L Y}$ & regressão & $\begin{array}{c}-0,0820^{* \cdots * *} \\
(-12,984)\end{array}$ & $\cdots$ & $\cdots$ & $\begin{array}{l}-0,0782^{* \ldots} \\
(-11,4360)\end{array}$ & $\cdots$ \\
\hline$\gamma_{F Y}$ & $-\gamma_{K Y}-\gamma_{E Y}-\gamma_{L Y}$ & $\begin{array}{l}-0,0050^{*} \\
(-1,320)\end{array}$ & $\cdots$ & $\cdots$ & $\begin{array}{l}-0,0052^{\mathrm{NS}} \\
(-1,0170)\end{array}$ & $\cdots$ \\
\hline$\gamma_{Y Y}$ & regressão & $\begin{array}{c}0,3146^{* * * *} \\
(9,705)\end{array}$ & $\begin{array}{c}0,2986^{* * * *} \\
(9,489)\end{array}$ & $\cdot \cdot$ & $\begin{array}{c}0,2959^{* * * *} \\
(8,0590)\end{array}$ & $\cdot \cdot$ \\
\hline$\gamma_{K X}$ & regressão & $\begin{array}{c}0,0916 \\
(7,382)\end{array}$ & $\begin{array}{l}0,0046^{\text {ns }} \\
(0,341)\end{array}$ & $\begin{array}{l}0,0062^{\text {ns }} \\
(0,450)\end{array}$ & $\cdot \cdot$ & $\cdots \cdot$ \\
\hline$\gamma_{E E}$ & regressão & $\begin{array}{c}0,0521^{* * * *} \\
(9,396)\end{array}$ & $\begin{array}{c}0,0529^{* * * *} \\
(9,615)\end{array}$ & $\begin{array}{c}0,0519^{* * * *} \\
(9,673)\end{array}$ & $\cdots \cdot$ & $\cdots \cdot$ \\
\hline$\gamma_{L L}$ & regressão & $\begin{array}{c}0,1729^{* * * *} \\
(13,621)\end{array}$ & $\begin{array}{c}0,1426^{* * * *} \\
(8,917)\end{array}$ & $\begin{array}{c}0,1334^{\text {t*n+* }} \\
(8,011)\end{array}$ & $\cdots$ & $\cdots$ \\
\hline$\gamma_{F F}$ & $-\gamma_{K F}-\gamma_{E F}-\gamma_{L F}$ & $\begin{array}{l}0,0155^{* * * * *} \\
(2,562)\end{array}$ & $0,0170^{* * * *}$ & $\begin{array}{l}0,0134^{4 * *} \\
(2,314)\end{array}$ & $\cdots$ & $\cdot \cdot \cdot$ \\
\hline$\gamma_{K E}$ & regressão & $\begin{array}{c}-0,0215^{* * * * * *} \\
(-4,340)\end{array}$ & $\begin{array}{c}-0,0217^{* * * *} \\
(-3,847)\end{array}$ & $\begin{array}{c}-0,0209^{* * * * *} \\
(-3,710)\end{array}$ & $\cdot \cdot$ & $\cdots \cdot$ \\
\hline$\gamma_{K L}$ & regressão & $\begin{array}{c}-0,0920^{* * * * 4} \\
(-8,180)\end{array}$ & $\begin{array}{l}-0,0308^{* * *} \\
(-2,309)\end{array}$ & $\begin{array}{l}-0,0289^{\prime * *} \\
(-2,111)\end{array}$ & $\cdots$ & $\cdots$ \\
\hline$\gamma_{K P}$ & $-\gamma_{K K}-\gamma_{K E}-\gamma_{K L}$ & $\begin{array}{c}0,0219^{* \cdots *} \\
(3,594)\end{array}$ & $\begin{array}{c}0,0479^{* * * * *} \\
(7,680)\end{array}$ & $\begin{array}{c}-0,0392^{* * * *} \\
(-6,792)\end{array}$ & $\cdots \cdot$ & $\cdots$ \\
\hline$\gamma_{E L}$ & regressão & $\begin{array}{c}-0,0372^{* \cdots * *} \\
(-8,105)\end{array}$ & $\begin{array}{c}-0,0390^{* * * *} \\
(-6,904)\end{array}$ & $\begin{array}{c}0,0437^{* * \ldots} \\
(6,896)\end{array}$ & $\cdots$ & $\cdots \cdot$ \\
\hline$\gamma_{E F}$ & $-\gamma_{K E}-\gamma_{E E}-\gamma_{E L}$ & $\begin{array}{c}0,0065^{* * *} \\
(1,597)\end{array}$ & $\begin{array}{c}0,0079^{* * *} \\
(1,996)\end{array}$ & $\begin{array}{c}0,0082^{* * *} \\
(2,043)\end{array}$ & $\cdots$ & $\cdots$ \\
\hline$\gamma_{L F}$ & $-\gamma_{K L}-\gamma_{E L}-\gamma_{L L}$ & $\begin{array}{c}-0,0438^{* * * *} \\
(-7,770)\end{array}$ & $\begin{array}{c}-0,0727^{* * * *} \\
(-10,862)\end{array}$ & $\begin{array}{c}-0,0653^{* \cdots *} \\
(-9,331)\end{array}$ & $\cdots$ & $\cdots$ \\
\hline Restriçסes & $\cdot$ & Nenhuma & (3) & (4) & $(6)$ & $(10)$ \\
\hline Log det $\Omega$ & - & $-21,196$ & $-20,589$ & $-20,286$ & $-20,230$ & $-19,428$ \\
\hline
\end{tabular}

Fonte: dados da pesquisa.

Nota: $\mathrm{O}$ valor da estatística $t$ (de student) encontra-se entre parênteses: ${ }^{* * *}$ significativo a $1 \%$; ${ }^{* * *}$ significativo a $5 \%$; "* significativo a $10 \%$; " significativo a $20 \%$; NS = Não significativo a $20 \%$. 
As estatísticas $t$ para os parâmetros de não homoteticidade $\left(\gamma_{\mathrm{Y}}\right)$ e elasticidade de substituição unitária $\left(\boldsymbol{\gamma}_{\mathbf{j}}\right)$ para o modelo A (translog) sugerem que, nem a hipótese de homoteticidade nem a de elasticidade de substituição unitária são consistentes com o conjunto de dados.

Esta suposição é confirmada pelo teste de "razão de verossimilhança" (likelihood ratio test), que tem seus resultados apresentados na Tabela 3. As estatísticas do teste de $\chi^{2}$ para os modelos B, C, D e E nos sugerem a rejeição estatística das hipóteses realizadas com respeito à função de produção de: homoteticidade, homogeneidade, elasticidade de substituição unitária e homogeneidade com elasticidade de substituição unitária.

Tabela 3: Teste de hipóteses para a imposição de homoteticidade, homogeneidade e elasticidade de substituição unitária à função de produção associada à equação de custo.

\begin{tabular}{lcccc}
\hline & \multicolumn{4}{c}{ Restrições impostas ao modelo A } \\
\cline { 2 - 5 } & $\begin{array}{l}\text { Homote- } \\
\text { ticidade }\end{array}$ & $\begin{array}{c}\text { Homogenei- } \\
\text { dade }\end{array}$ & $\begin{array}{c}\text { Elasticidade } \\
\text { de } \\
\text { substituição } \\
\text { unitária. }\end{array}$ & $\begin{array}{c}\text { Homogeneidade } \\
\text { e elasticidade de } \\
\text { substituição unitária. }\end{array}$ \\
\hline $\begin{array}{l}\text { Restrições } \\
\text { impostas }\end{array}$ & $\gamma_{i Y}=0$ & $\begin{array}{c}\gamma_{i Y}=0 \\
\gamma_{Y Y}=0\end{array}$ & $\gamma_{i j}=0$ & $\gamma_{i Y}=0$ \\
$\gamma_{Y Y}=0$ e $\gamma_{i j}=0$ \\
$\begin{array}{l}\text { Total de } \\
\text { restrições (Graus } \\
\text { de Liberdade) } \\
\begin{array}{l}\text { Valor calculado } \\
\text { do } \chi^{2}\end{array}\end{array}$ & 3 & 4 & 6 & 10 \\
\hline
\end{tabular}

Fonte: dados da pesquisa.

Nota: "significativo a $1 \%$

Desta forma, pode-se concluir que o modelo de custos translog (modelo A) - que permite não homoteticidade, não homogeneidade e elasticidades de substituição não unitária à estrutura de produção associada - é o mais adequado para representar a estrutura de custos do setor de produção de frangos de corte no Brasil. Verifica-se, assim, para este modelo, as condições que a função custo têm que satisfazer para corresponder a estruturas de produção bem-comportadas de, monotonicidade e convexidade nos preços dos fatores. 
A monotonicidade da função é comprovada pelo comportamento das parcelas de custos, calculadas pela média aritmética da amostra, que se apresentam todas positivas: $\mathrm{SK}=0,4083$; $\mathrm{SE}=0,1685$; $\mathrm{SL}=0,3346$ e $\mathrm{SF}=0,0886$.

Os sinais das elasticidades-preço diretas da demanda por fatores $\left(\eta_{i i}\right)$, bem como os das elasticidades de substituição parcial de Allen diretas $\left(\sigma_{i i}\right)$, foram todos negativos (Tabela 4$)$, o que significa que a concavidade da função custos também foi atendida.

Tabela 4: Estimativa das elasticidades-preço diretas $\left(\eta_{i i}\right)$ e de substituição parcial de Allen, diretas entre os fatores de produção $\left(\sigma_{i i}\right)$, para o modelo A de custos translog.

\begin{tabular}{ccccc}
\hline & Capital & Energia & Trabalho & Cama de frango \\
\hline$\eta_{\boldsymbol{i i}}$ & $-0,3672$ & $-0,5221$ & $-0,1485$ & $-0,7370$ \\
& $(-12,08)$ & $(-15,86)$ & $(-3,914)$ & $(-10,83)$ \\
$\sigma_{i i}$ & $-0,8993$ & $-3,0985$ & $-0,4439$ & $-8,3228$ \\
& $(-29,58)$ & $(-94,12)$ & $(-11,70)$ & $(-122,3)$ \\
\hline
\end{tabular}

Fonte: dados da pesquisa.

Nota: O valor da estatística $t$ (de student) encontra-se entre parênteses: todos os coeficientes significativos a $1 \%$.

\section{2 Índices de economias de escala para a amostra de produtores}

Estimativas de economias de escala (IES) podem ser calculadas para cada produtor individualmente, ao nível observado de produção e preços de fatores, através das fórmulas da Tabela 1. Adota-se, no entanto, o procedimento descrito em Christensen \& Greene (1976) e divide-se a amostra dos produtores em grupos, de acordo com o nível de produção. Optou-se por dividir a amostra em seis grupos. Os IES são calculados para a média de produção e preços de cada grupo como segue: 1) produção média de 18,58 mil quilos de frango vivo; 2) 37,16 mil quilos; 3 ) 55,74 mil quilos; 4) 92,89 mil quilos; 5) 198,16 mil quilos e 6) produção média de 297,24 mil quilos. Na Tabela 5 apresentam-se os resultados dos IES para a média dos grupos. A fim de possibilitar comparações, são calculados os IES para todos os modelos considerados. 
Os IES para os modelos C e E - que impõem homogeneidade à função de produção - são constantes para todos os níveis de produto. Segundo Christensen \& Greene (1976), este resultado deixa a errônea impressão que há economias de escala significativas para todos os tamanhos de planta. Não obstante, os modelos não homogêneos indicam que as economias de escala se esgotam dentro dos níveis de produção da amostra, passando algumas plantas a apresentarem deseconomias. Para os modelos A, B e D observa-se que, como esperado, as economias de escala diminuem com o aumento da produção. As estimativas de IES para estes modelos são muito similares. O efeito da imposição de homoteticidade sobre a função custos translog do modelo A faz com que os IES sejam ligeiramente superiores, enquanto a imposição de elasticidade de substituição unitária reduz o índice.

Tabela 5: Índices de economias de escala (IES) para diferentes níveis de produção de frangos de corte no Brasil.

Nível de produção (em $1.000 \mathrm{~kg}$ de frango vivo)

\begin{tabular}{lcccccc} 
& 18,58 & 37,16 & 55,74 & 92,89 & 198,16 & 297,24 \\
\hline Modelo A & 0,53 & 0,33 & 0,19 & 0,04 & $-0,17$ & $-0,30$ \\
Modelo B & 0,61 & 0,40 & 0,28 & 0,19 & $-0,10$ & $-0,22$ \\
Modelo C & 0,29 & 0,29 & 0,29 & 0,29 & 0,29 & 0,29 \\
Modelo D & 0,52 & 0,33 & 0,19 & 0,04 & $-0,15$ & $-0,27$ \\
Modelo E & 0,25 & 0,25 & 0,25 & 0,25 & 0,25 & 0,25 \\
\hline
\end{tabular}

Fonte: dados da pesquisa.

Para o modelo de custos translog (A), verifica-se que produtores com nível de produção de 198,16 mil quilos apresentam deseconomias de escala bastante acentuadas, que se agravam quando a produção aumenta para 297,24 mil quilos.

É interessante verificar os resultados encontrados para os IES do modelo A, considerando-se o tamanho da planta, em $\mathrm{m}^{2}$ (metro quadrado) de aviário, que corresponde à produção média especificada. Dado um peso médio de 2,3Kg e uma densidade média de 13,7 frangos por metro quadrado, tem-se para os estratos considerados o equivalente 
a $600 \mathrm{~m}^{2}$ de capacidade de alojamento na propriedade para o primeiro estrato, $1.200 \mathrm{~m}^{2}$ para o segundo, $1.800 \mathrm{~m}^{2}$ para o terceiro, $3.000 \mathrm{~m}^{2}$ para o quarto estrato, $6.400 \mathrm{~m}^{2}$ para produção do quinto estrato e $9.600 \mathrm{~m}^{2}$ para o sexto e último estrato. Assim, conforme sugerem os resultados do modelo estimado, verifica-se que propriedades com $6.400 \mathrm{~m}^{2}$ de aviário ou metragem superior apresentam deseconomias de escala, dentro da amplitude da amostra.

\subsection{Curva de custo médio para a amostra de produtores de frango}

Utilizam-se os parâmetros do modelo estimado para construir a curva de custo médio da amostra de dados (Figura 1). A curva de custo médio é derivada avaliando-se sua função para uma gama de produções, mantendo-se os preços dos fatores fixos na média da amostra.

Figura 1: Curva de custo médio para a produção de frangos de corte no Brasil - Modelo Translog.

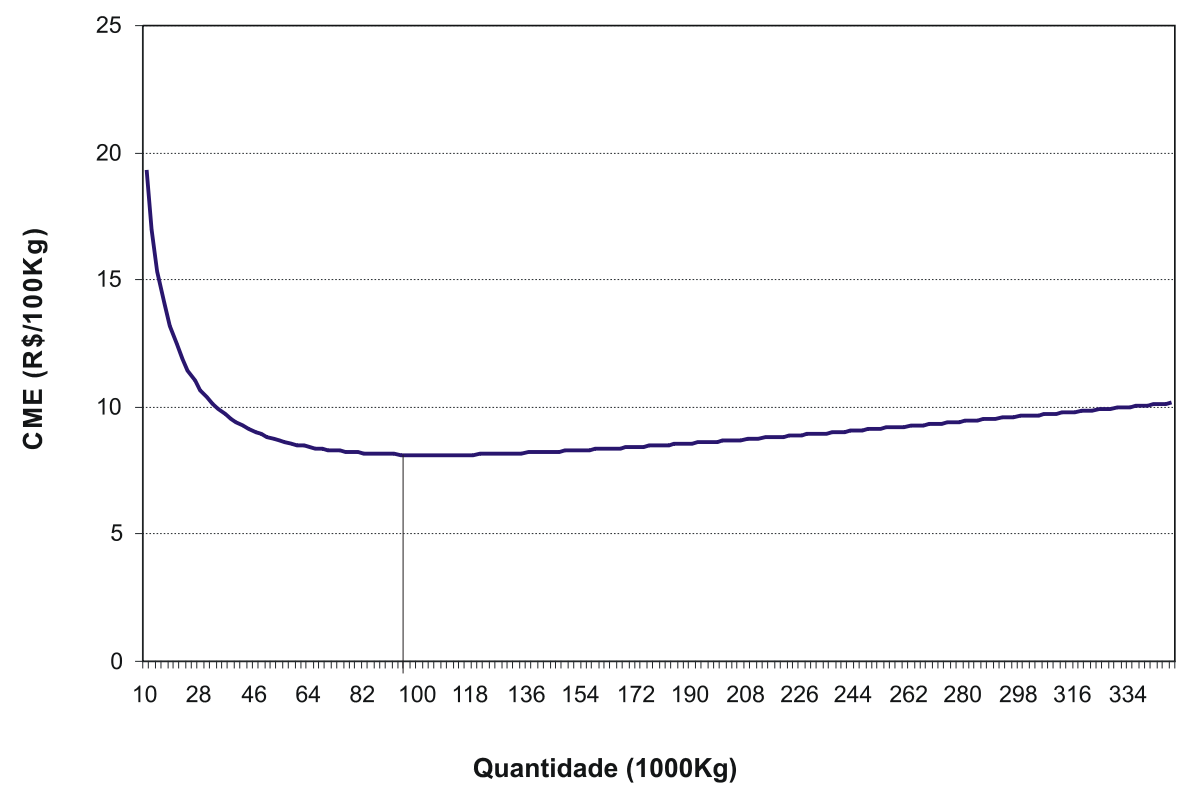

Fonte: Dados da pesquisa 
Observa-se que a curva de custo médio correspondente ao modelo translog, apresenta a forma clássica de $\mathrm{U}$, com os custos decrescendo com o nível de produção até um ponto de mínimo, quando então os custos médios passam a ser crescentes. A escala ótima de produção ocorre quando se obtém retornos constantes à escala, ou seja, quando o produtor está operando no ponto de custo mínimo da curva de custo médio. Além deste ponto tem-se deseconomias e o produtor passa a operar no ramo crescente da curva de custo médio.

Observa-se, pela Figura 1, que o ponto de mínimo custo corresponde a uma produção de aproximadamente 110 mil quilos de frango vivo. Considerando-se mais uma vez o peso médio de $2,3 \mathrm{~kg}$ e uma densidade de aproximadamente 13,7 aves por $\mathrm{m}^{2}$, tem-se a escala ótima de produção em propriedades que possuem $3.500 \mathrm{~m}^{2}$ de aviário aproximadamente. Além desta capacidade de produção, haveria deseconomias de escala.

\section{Conclusões}

A amostra de produtores de frango pesquisada representou bem as realidades regionais, com o predomínio de produtores com pequenas propriedades e menor capacidade produtiva no Sul do país e Minas Gerais e produtores com propriedades maiores e níveis de produção mais elevados nos estados do Centro-Oeste brasileiro. Nas regiões Oeste do Paraná e no Triângulo Mineiro (regiões tradicionais produtoras de soja e milho) encontramos os produtores com maiores áreas de terra e com as maiores capacidades de alojamento de aves da amostra da região tradicional. No Centro-Oeste, os produtores visitados no Estado do Mato Grosso do Sul foram os que apresentaram menores áreas de terra e menores estratos de capacidade de produção de frangos de corte, enquanto os produtores pesquisados do Estado de Goiás foram os que apresentaram os maiores estratos de capacidade de produção da região.

Verificou-se, na pesquisa de campo realizada, que $24,7 \%$ das propriedades da amostra da região Sul e Minas Gerais possuem até $840 \mathrm{~m}^{2}$ de aviário e $63,1 \%$ até $1.350 \mathrm{~m}^{2}$; com possibilidades, portanto, de redução do custo médio com a ampliação do nível de operação, aproveitando as economias de escala disponíveis. Destaca-se que, nessa região, apenas 6,8\% dos produtores pesquisados possuíam de $3.000 \mathrm{~m}^{2}$ a $3.700 \mathrm{~m}^{2}$ de aviários 
na propriedade (bem próximo, portanto, do ponto limite de esgotamento das economias de escala) e apenas $2 \%$ tinham mais de $3.700 \mathrm{~m}^{2}$ de aviário (trabalhando, portanto, na faixa das deseconomias de escala).

A amostra pesquisada poderia sugerir que, na região tradicional, a ampliação da produção na maioria das propriedades poderia levar a um melhor aproveitamento das economias de escala. Conclui-se porém, com base na amostra, que há na região tradicional, dificuldades na ampliação da produção na grande maioria das propriedades produtoras, dado os fatores limitantes de área e mão-de-obra. Uma exceção seria a região Oeste do Paraná e na região do Triângulo Mineiro, nas quais encontramos avicultores com áreas de terra maiores.

Na região Centro-Oeste, por sua vez, $24,1 \%$ da amostra dos produtores pesquisados possuem de 900 a $1.350 \mathrm{~m}^{2}$ de aviários na propriedade, $15,7 \%$ de 1.400 a $2.000 \mathrm{~m}^{2}, 21,7 \%$ de 2.100 a $2.700 \mathrm{~m}^{2}, 10,8 \%$ de 3.000 a $3.700 \mathrm{~m}^{2}$ e $20,5 \%$ de 6.000 a $6.400 \mathrm{~m}^{2}$. No total da amostra da região $72,3 \%$ dos produtores pesquisados tem até $3.700 \mathrm{~m}^{2}$ de aviários na propriedade. Isto significa que $27,7 \%$ da amostra de produtores da região trabalham na faixa das deseconomias. Operando com rendimentos decrescentes de escala, estes produtores poderiam exercer uma certa pressão por melhores remunerações para a atividade, por parte da agroindústria parceira. Além do mais, a própria estrutura da produção em parceria, implantada nos novos projetos do Centro-Oeste brasileiro, composta por um pequeno número de grandes produtores, expõe a agroindústria e fortalece os produtores, em termos de negociação de preços.

Destaca-se que, no cálculo dos índices de economia de escala encontrados, leva-se em conta os custos de produção que efetivamente são do produtor, por força de contrato. Não sendo incluídos, portanto, os custos com ração e pintos de um dia e os seus correspondentes custos de transporte, que são de responsabilidade da agroindústria. Acredita-se que, se estes custos fossem de responsabilidade do produtor, os resultados encontrados poderiam indicar economias de escala significativas para plantas ainda maiores, dado que, teoricamente, ter-se-iam ganhos em termos de redução de custos, devido às distâncias maiores na região produtora do Centro-Oeste brasileiro. Cabe observar, também, que os maiores produtores de frangos do Centro-Oeste, que fizeram altos investimentos em instalações de aviários, receberam preços mais elevados por 
ave produzida do que os estratos menores da região tradicional, o que pode ter compensado, em parte, as perdas decorrentes do tamanho.

As empresas, na região de expansão, estimulam a produção em escalas maiores que a ótima estimada (em um dos projetos avícolas implantados na região, a empresa opta por núcleos de produção com até $6.400 \mathrm{~m}^{2}$ de aviário), muito provavelmente devido às distâncias relativamente maiores entre as propriedades e a agroindústria. Núcleos de produção maiores facilitariam a logística de assistência técnica e veterinária, bem como do abastecimento de ração e pintos de um dia. Por outro lado, os avicultores entram no sistema de parceria com a agroindústria fazendo os investimentos necessários à produção em núcleos maiores que o ótimo, convencidos de uma rentabilidade que seja satisfatória para a atividade e compensem os investimentos. Além disto, outros incentivos atraem os produtores da região Centro-Oeste para o sistema de produção em parceria avícola, nas escalas sugeridas pelas agroindústrias: primeiro, a facilidade (como no caso dos novos projetos avícolas) de aquisição do capital financeiro necessário à montagem dos núcleos produtivos, com garantias da agroindústria parceira e com prazos e juros atrativos; segundo, a possibilidade de utilização do resíduo orgânico da atividade (cama de frango) na adubação das plantações da região.

Avalia-se que os produtores são atraídos e adquirem estas linhas de crédito para instalação dos núcleos produtivos, dado que os custos de oportunidade para a aquisição do capital são quase nulos. Ou seja, os recursos e as condições de captação dos mesmos são específicos para a entrada na atividade. Assim o valor real do custo do capital fica "pouco visível" ao produtor. É importante destacar que, os custos serão mais visíveis (portanto melhor avaliados), quanto maior o seu custo de oportunidade.

Os resultados empíricos sugerem que as pequenas propriedades da região tradicional não estão aproveitamento todas as economias de escala possíveis. Apesar disso, alguns fatores, dentre outros, são determinantes para minimizar esta desvantagem em relação à maior produção e para a manutenção destas propriedades na atividade: (1) a longa experiência dos produtores na atividade, que se reflete em um melhor manejo na produção; (2) o fato, captado na pesquisa, da renda proveniente da atividade de produção de frangos representar um percentual significativo da renda total do proprietário. Esta questão geraria uma certa subordinação do pe- 
queno produtor a agroindústria, o que ocasionaria uma predisposição de aceitar remunerações menores pelo lote de frango; (3) a maior densidade de indústrias e de produtores na região tradicional. O investimento em plantas industriais de abate e em aviários é elevado. Dado a especificidade dos ativos produtivos, os custos de saída da atividade são, da mesma forma, altos (tanto para o produtor quanto para a indústria); (4) devido à grande densidade de pequenos produtores produzindo em pequenas propriedades, as agroindústrias parceiras tem facilidade no planejamento logístico da distribuição da ração e dos pintos de um dia, bem como da assistência técnica e veterinária, que são feitos por áreas de produção; (5) os sistemas cooperativos, consolidados na região, que incentivam a atividade e, em alguns casos, remuneram melhor o produtor, inclusive com distribuição de sobras financeiras; (6) a proximidade, da região produtora tradicional, dos portos marítimos, dado que uma significativa parcela da produção dos estados do Sul e de MG é destinada à exportação.

\section{Referências bibliograficas}

ALBUQUERQUE, M.C.C. de. Uma análise translog sobre mudança tecnológica e efeitos de escala: Um caso de modernização eficiente. Pesquisa e Planejamento Econômico, v.1, n.17, p.191-220, abr. 1987.

ALVES, E. A função Custo. Brasília: EMBRAPA-SPI, 1996. 106p.

BAIRAM, E.I. (Ed.). Production and cost functions: Specification, measurement and applications. Vermont: Ashgate publishing ltd, 1998. 132p.

BARBOSA, F. de H. Microeconomia: Teoria, modelos econométricos e aplicações à economia brasileira. Rio de Janeiro: IPEA/INPES, 1995. $556 \mathrm{p}$.

BERNDT, E.R.; WOOD D.O. Thechnology, prices and the derived demand for energy. The Review of Economics and Statistics, v.LVII, n.3, p.259268, Aug. 1975.

BINSWANGER, H.P. A cost function approach to the measurement of elasticities of factor demand and elasticities of substitution. American Journal of agricultural Economics, v.56, p.377-386, May. 1974. 
BUARQUE, C. Avaliação econômica de projetos. Rio de Janeiro: Campus, 1991. 266p.

CANEVER, M.D. Competitividade relativa entre as cadeias de carne de frango Brasileira e Argentina. Viçosa, 1997. 134p. Dissertação (Mestrado) - UFV.

CHAMBERS, R.G. Applied production analysis: A dual approach. New York, Cambridge University Press, 1994. 331p.

CHRISTENSEN, L.R.; GREENE, W.H. Economies of scale in U.S. electric power generation. Journal of Political Economy, v.84, n.4, p. 655-676, 1976.

FERREIRA, A.A. Características dos sistemas de produção, eficiência econômica e economias de escala na produção de frangos de corte no Estado de Minas Gerais. Viçosa, 1998. 140p. Dissertação (Mestrado) - UFV.

GREENE, W.H. Econometric Analysis. 4th ed. New Jersey: PrenticeHall, 2000. 1.004p.

GARCIA, L. A. F. Economias de escala na produção de frangos de corte no Brasil. Piracicaba, 2004. 114p. Tese (Doutorado) - Escola Superior de Agricultura "Luiz de Queiroz", Universidade de São Paulo.

HOFFMANN, R.; ENGLER, J.J. de C.; SERRANO, O. et al. Administração da empresa agrícola. $2^{\text {a }}$ ed. São Paulo: Pioneira, 1987. 325p.

LERDA, J.C. Resultados básicos na teoria da dualidade: vantagens e alguns usos em microeconomia. Estudos Econômicos, v.9, n.1, p.101-133, jan./abr. 1979.

MARTINS, S.S. Situação e perspectivas da avicultura paulista. Informações Econômicas, v.32, n.6, p.74-77, Jun. 2002.

RAY, S.C. A translog cost function analysis of U.S agriculture, 193977. American Journal of Agricultural Economics, v.56, p: 490-498, 1982.

REIS, R.P.; TEIXEIRA, E.C. estrutura de demanda e substituição de fatores produtivos na pecuária leiteira: o modelo de custo translog. Revista Brasileira de Economia, v.49, n.3, p.545-554, jul./set. 1995. 
ROCHELLE, T.C.P.; FERREIRA FILHO, J.B. de S. Função custo translog e o mercado de fatores para o algodão no Estado de São Paulo: O caso da DIRA de Campinas. Revista de Economia e Sociologia Rural, v.37, n.2, p.77-95, abr./jun. 1999.

SILBERBERG, E. The structure of economics: A mathematical analysis. $2^{\mathrm{a}}$ ed. New York: Mc Graw - Hill Inc. 1990. 686p.

ZAGATTO, L.C.A.G. Estrutura produtiva de pequenos agricultores e implicações para a geração e adoção de tecnologia. Viçosa, 1991. 112p. Tese (Doutorado) - UFV.

ZELLNER, A. An efficient method of estimating seemingly unrelated regressions and tests for aggregatton bias. Journal of the American Statistical Association, n.57, p.348-368, Jun. 1962.

Recebido em agosto de 2004 e revisto em agosto de 2005 